\title{
Assessment of Offshore Pipeline Reliability against Lateral Buckling
}

\author{
Seyed Mohammad Hossein Sharifi ${ }^{*}$, Abdolrahim Taheri², Mohammad Bagher Faraji Pool ${ }^{3}$ \\ ${ }^{1}$ Assistant Professor, Department of Mechanical Engineering, Petroleum University of Technology, Iran; \\ sharifi@put.ac.ir \\ ${ }^{2}$ Assistant Professor, Department of Mechanical Engineering, Petroleum University of Technology, Iran; \\ rahim.taheri@put.ac.ir \\ ${ }^{3}$ M.Sc. Student, Petroleum University of Technology, Iran; m.farajipool@mnc.put.ac.ir
}

\section{ARTICLE INFO}

\section{Article History:}

Received: 28 Jan. 2019

Accepted: 21 Sep. 2019

Keywords:

Subsea Pipeline

Lateral Buckling

Corrosion

Reliability

Probability of failure

\begin{abstract}
Subsea pipelines are used to transport gas and oil around the world. Oil is transported through subsea pipelines at high pressure and high temperature to smooth the way for its flow and to prevent its solidification. The present paper assesses a pipeline located in South Pars Gas Field against lateral buckling. As more and more pipelines operate at higher temperatures (over $100^{\circ} \mathrm{C}$ ), the likelihood of lateral buckling becomes more relevant. The uncertainty in the lateral buckling parameters of the pipeline is a source of error in determining effective axial compressive force. Uncontrolled lateral buckling can cause excessive plastic deformation of the pipeline, which can lead to localized buckling collapse or cyclic fatigue failure during operation due to multiple heat-up and cool-down cycles, if it is not properly managed. This research reports the results of a reliability analysis to study and quantify the variations of the reliability index $(\beta)$ with the main parameters involved during the lateral buckling of the subsea pipelines. Uncertainty is considered in the geometric parameters of the pipeline. The probability of failure $\left(\mathrm{P}_{\mathrm{f}}\right)$ and the reliability index $(\beta)$ can be determined by the reliability methods. The First-Order Reliability Method (FORM), the Second-Order Reliability Method (SORM) and the sampling method are the three main methods used here to determine $P_{f}$ and $\beta$. The results show that the pipelines, in the case of lateral buckling and corrosion, will be in safe condition for up to 30 years after construction.
\end{abstract}

\section{Introduction}

Submarine pipelines are used for various purposes in the improvement of subsea hydrocarbon resources. Offshore pipelines are one of the most effective tools for transporting hydrocarbon productions from wells to terminals/platforms located on the shore or offshore $[1,2]$. As offshore industries go for deeper resources, pipelines should be checked for resistance against new loads in subsea conditions [3]. Due to the substantial uncertainty in new environments and also in operating conditions of the pipelines, the need for the use of reliability-based methods greatly increases. To measure the reliability of a system, the system is first broken down into components, and the reliability of the system is expressed in terms of the reliability of its components. To calculate the reliability of each component based on available statistical data, a model for the failure rate is selected and its parameters are estimated by the available data. Given the uncertainty in the geometric parameters of a structure and the environmental conditions, the reliability assessment methods show the probability of failure of the structure under special loading conditions. Uncertainties affecting the safety of marine structures, such as drought structures, may occur both in loading and in the strength of the structural components and fittings. Marine structures should be safe and stable in different loading conditions.

Buckling is one of the ultimate limit state failure modes affecting maintenance costs. Normally, buckling may happen in two modes: local and global. Local buckling happens due to the out-of-roundness and global buckling is associated with high-temperature/highpressure gradient along submarine pipelines. Based on the buckle plane, global buckling can occur in the horizontal and vertical directions, which are called lateral and upheaval buckling, respectively [4, 5]. Furthermore, lateral and upheaval buckling may happen for on-bottom and buried pipelines, respectively. 
If a pipeline is not free to expand in the operation, restrained axial deformation generates an axial compressive force in the pipeline. The pipeline usually is not perfectly straight with some out-of- straightness (OOS), and the imperfections are typically due to the pipeline being laid over irregularities in the seabed profile. The phenomenon of lateral buckling has widely been investigated over the past decades. Many methods have been adopted over the years to mitigate the lateral buckling, including snake lay, sleepers, distributed buoyancy, trench and bury, and expansion spools. Planned buckle initiators such as distributed buoyancy sections or sleeper pipe upsets are often designed to manage the global buckling to ensure pipeline integrity. They have successfully been used in many projects. The planned buckle initiators are spaced periodically along the pipeline to alleviate the axial load down to an acceptable level. In recent years, finite element analysis software has extensively been employed in simulation and prediction of pipeline responses to global buckling. A research paper on the on-bottom stability of the pipeline under the influence of wave and current load uses a nonlinear finite element model to compute combined stresses/lateral displacement acting on offshore pipelines due to combined hydrodynamic loads including wave/current effects. The model takes into account the effect of pipe-soil interaction. The resulting combined pipe stresses/lateral displacement is used as input for the reliability assessment. Three case studies for actual offshore pipelines of Egypt have been analyzed using the proposed approach. The results show that the computed safety levels are within the target values. The proposed approach can be a valuable tool for pipeline designers/operators for the assessment of the pipeline safety and reliability with respect to onbottom stability [6].

In his thesis on the assessment of the system reliability of offshore pipelines, Mostafa has identified, applied, and judged on the suitability of a probabilistic method for evaluating the reliability of an offshore pipeline system against corrosion [3].

Two joint industry projects (JIP), namely HOTPIPE and SAFEBUCK, were recently implemented to develop industry knowledge of the design of HPHT pipelines susceptible to global buckling [4].

HOTPIPE JIP [7] is a strategy for the structural design of HPHT pipelines. The design criteria are based on the application of the reliability methods to calibrate the partial safety factors in compliance with the safety philosophy established by DNV-OS-F101.

The SAFEBUCK JIP-SAFEBUCK design guideline [8] proposes a methodology, based on in-place survey data of four operational pipelines donated by the JIP members.

If the compressive effective axial force is large enough, slender structures, such as pipelines, will undergo Euler buckling (global buckling). The global buckling includes lateral buckling and upheaval buckling. The typical lateral friction coefficients are smaller than unity, so the uniformly distributed lateral friction force generated by soil resistance is smaller than the submerged weight of a pipeline. A pipeline laid onto the seabed without trenching or cover tends to create lateral buckles rather than upheaval buckles. The problem of lateral buckling in pipelines was addressed and theoretically analyzed by Hobbs and Liang in early research [9].

The DNV-OS-F101 [10] gives criteria and recommendations on conceptual development, design, construction, operation, and abandonment of submarine pipeline systems and the DNV-RP-F110 [7] is the common industry recommended practice for designing submarine pipelines against global buckling. The DNV uses the mean values of soil resistance and driving force (e.g. on effective axial load) in the design process as a deterministic method. The variability in soil resistance and force is reduced by applying the load factor $(\gamma U F)$ on the driving force. Appropriate values should be used for partial safety factors $(\gamma U R, \gamma U F)$ in the design phase to increase the safety, and the factors depend on the accuracy of field measurements and the targeted safety class. This conventional deterministic method is simple and straightforward but does not take the variability into account appropriately. These aspects can be examined by a probabilistic approach considering the variability in the inputs and assessing their effects on the overall global buckling behavior. On the other hand, in a probabilistic approach, the input parameters and loading are regarded as continuous random variables and the performance of the structure resulting from different failure criteria is expressed in a probabilistic framework as the probability of failure $\left(P_{f}\right)$ and/or the reliability index $(\beta)$ [11].

This paper investigates the effect of variability in pipe properties, e.g. thickness, diameter, and elastic modulus of the pipeline on the reliability index in lateral buckling.

\section{Case Study}

The studied steel pipeline has a diameter $(D)$ of 0.6156 $\mathrm{m}$ (24.24 in), a thickness of $0.024 \mathrm{~m}$ and a length of 40 $\mathrm{m}$. Poisson's ratio $(\vartheta)$ of the pipe is assumed to be equal to 0.3 and the coefficient of thermal expansion $\left(\alpha_{t}\right)$ is equal to $11.5 \times 10^{-6}\left[{ }^{\circ} \mathrm{C}^{-1}\right]$. The distribution of the undrained shear strengths was determined to be lognormal using field data. The reasons for neglecting the residual tension are that those axial forces are generally associated with a high degree of uncertainty and their influence is very case-specific [12]. Pipeline submerged weight in operational conditions is equal to $4293(\mathrm{~N} / \mathrm{m})$. Table 1 presents the general parameters of the pipelines. 


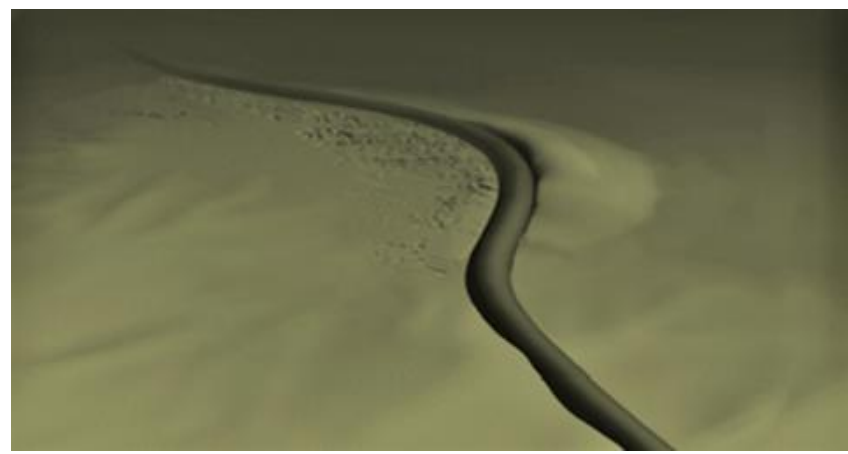

Figure 1. Schematic diagram of lateral buckling in offshore pipeline [13]

Table 1. The properties of the pipeline [12]

\begin{tabular}{ll}
\hline Characteristic & Value \\
\hline Pipeline outside diameter & $0.6156[\mathrm{~m}]$ \\
\hline Steel pipeline wall thickness & $0.024[\mathrm{~m}]$ \\
\hline Steel pipeline density & $7850\left[\mathrm{~kg} / \mathrm{m}^{3}\right]$ \\
\hline Modulus of elasticity & $210[\mathrm{GPa}]$ \\
\hline Poisson's ratio & $0.3[-]$ \\
\hline Thermal expansion coefficient & $11.5 \times 10^{-6}\left[{ }^{\circ} \mathrm{C}^{-1}\right]$ \\
\hline Pipeline submerged weight in & $4293[\mathrm{~N} / \mathrm{m}]$ \\
operation condition & \\
\hline $\begin{array}{l}\text { Difference between operating } \\
\text { and installation temperature }\end{array}$ & $75[\mathrm{C}]$ \\
\hline Operation pressure & $10.85[\mathrm{MPa}]$ \\
\hline Maximum water depth & $85[\mathrm{~m}]$ \\
\hline Seawater density & $1023\left[\mathrm{~kg} / \mathrm{m}^{3}\right]$ \\
\hline
\end{tabular}

Table 2. Uncertainties of parameters with their relevant mean and C.O.V [12]

\begin{tabular}{lllll}
\hline Row & Parameter & $\begin{array}{l}\text { Distribution } \\
\text { Type }\end{array}$ & Mean & C.O.V \\
\hline 1 & $\begin{array}{l}\text { Young's } \\
\text { modulus }\end{array}$ & Log-normal & $210 \times 10^{9}$ & 0.05 \\
\hline 2 & $\begin{array}{l}\text { Pipeline wall } \\
\text { thickness }\end{array}$ & Normal & 0.024 & 0.05 \\
\hline 3 & $\begin{array}{l}\text { Pipeline } \\
\text { diameter }\end{array}$ & Normal & 0.6156 & 0.05 \\
\hline
\end{tabular}

\section{Analytical Solution of lateral Buckling}

The parameters and equations used to determine the lateral buckling are presented below [14]. The required effective axial force to buckle can be expressed as [4]

$P_{(z)}=\frac{k_{1} \cdot E \cdot I}{[L(z)]^{2}}+k_{3} \cdot \mu_{a} \cdot \omega \cdot L_{(z)}$.

$\left\{\left[1+\frac{k_{2} \cdot E \cdot A \cdot \mu_{l}^{2} \cdot \omega \cdot\left(L_{(Z)}\right)^{5}}{\mu_{a}(E \cdot I)^{2}}\right]^{0.5}-1\right\}$

The buckle amplitude is $y_{(z)}=\frac{k_{4} \cdot \mu_{l} \cdot \omega \cdot[L(z)]^{4}}{E \cdot I}$

The force left in the buckle is

$P_{\text {buck (z) }}=\frac{k_{1} \cdot E . I}{[L(z)]^{2}}$

The maximum moment induced in the buckle is

$M=k_{5} \cdot \mu_{l} \cdot \omega \cdot[L(z)]^{2}$

The values of buckling constants $k_{n}$ in these equations are given in Table 3 .

Lateral buckle shapes are difficult to predict due to the complicated soil-pipe interactions, random out-ofstraightness features, and the inherent instability of buckling behavior. But, the mode 3 happens more than other mode shapes in the lateral buckling. Therefore, the values of the mode 3 were considered for $k_{n}$.
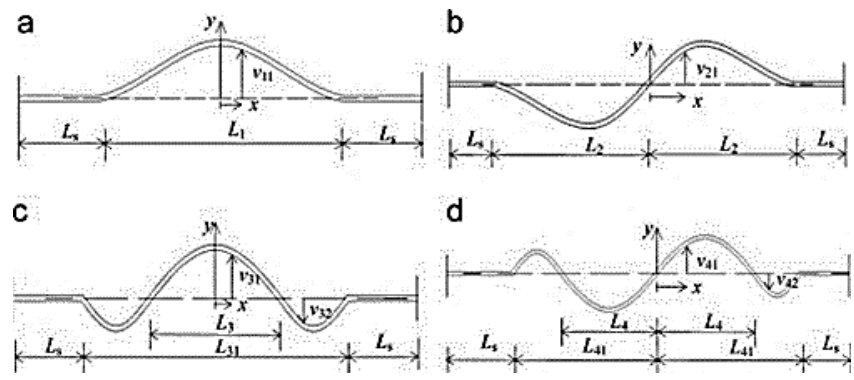

Figure 2. Buckling mode shapes [4]

Table 3. Buckling constants $\boldsymbol{k}$ for buckling modes [4]

\begin{tabular}{llllll}
\hline Buckle mode & $\boldsymbol{k}_{\mathbf{1}}$ & $\boldsymbol{k}_{\mathbf{2}}$ & $\boldsymbol{k}_{\mathbf{3}}$ & $\boldsymbol{k}_{\mathbf{4}}$ & $\boldsymbol{k}_{\mathbf{5}}$ \\
\hline 1 & 80.76 & $6.39 \mathrm{e}-5$ & 0.5 & $2.41 \mathrm{e}-3$ & 0.0694 \\
\hline 2 & 39.48 & $1.74 \mathrm{e}-4$ & 1.0 & $5.53 \mathrm{e}-3$ & 0.1088 \\
\hline 3 & 34.06 & $1.67 \mathrm{e}-4$ & 1.29 & $1.03 \mathrm{e}-2$ & 0.1434 \\
\hline 4 & 28.20 & $2.14 \mathrm{e}-4$ & 1.61 & $1.05 \mathrm{e}-2$ & 0.1483 \\
\hline
\end{tabular}

If a pipeline is subjected to buckle, the development of the effective force is modified as the pipe feeds into the buckle. The force in the buckle drops as the buckle develops. The maximum amount of pipe that can be fed into a buckle is equal to the unrestrained expansion of the line. However, the axial force in the buckled section $(P)$ would not be zero. The increase in the length of the pipe $\Delta l$ in the buckled section from the unbuckled state can be determined as

$\Delta l=\frac{\left(P_{\text {buck }}-P\right) \cdot L}{A \cdot E}$

The global response of a pipeline is determined by the compressive effective axial force. For a fully restrained pipeline, the effective axial force is expressed as

$S_{0}=F_{\text {residual }}-(1-2 v)\left(\Delta P_{i}\right) A_{i}-E A_{S} \alpha\left(\Delta T_{i}\right)(6)$

Lateral buckling is governed by three parameters: effective axial force, out-of-straightness, and boundary restraint [4]. 


\section{Reliability Assessment}

The reliability assessment accounts for the inevitable variability in pipe properties (geometry and material strength) which is the result of the normal perturbations in manufacturing processes used to produce the pipe. Variability in pipe properties produces uncertainty in collapse resistance, which can be addressed and managed by the reliability assessment.

The collapse pressure limit state depends on the pipe dimensions (diameter, ovality, and wall thickness), and material strength properties (stress-strain curve in the hoop and axial directions). Therefore, it is necessary to develop appropriate Probability Density Functions (PDFs) that characterize the expected statistical variations in these geometric and material properties for the use in the reliability analysis.

In the present context, the reliability is defined as the probability that a certain length of a pipe will not collapse due to a combination of external pressure and bending loads during construction or operation. The reliability is equal to the probability of failure subtracted from unity [15].

\section{Analysis Methodology}

The reliability of the lateral buckling design schemes has been developed using the structural reliability analysis (SRA) techniques reported in several papers, [16-18]. SRA methods are adopted to rationally treat the various sources of uncertainty involved in the buckling analysis. The probability of buckling initiation is calculated as

$P_{f}=$ Probability $[Z \leq 0]$

where $P_{f}$ is the probability of failure and $Z$ is the limit state function describing the buckle formation, which is obtained by recasting the buckling formation criteria. Eq. (8) denotes the buckling limit state function:

$Z=P_{(z)}-S_{0}$

The probability of buckling failure is determined by using first- and second-order reliability methods and the Monte Carlo method.

Since the basic random variables are modeled by continuous probability functions and the failure probabilities are small, it is preferable to apply analytical first- and second-order reliability methods (FORM and SORM, respectively). These methods are very efficient and accurate for small failure probability problems, FORM is of particular interest when the limit state function is relatively simple (e.g. expressed analytically).

\subsection{FORM}

For the nonlinear limit state surface, the FORM uses a linear approximation to the limit state at the design point and estimates the probability of failure as Eq. (13). If all the variables are not normally distributed, as is common in structural problems, it will be difficult to relate $\beta$ to the exact probability of failure.

Considering the function $(T)$ to transfer the input data $(X)$ to the standard normal space $(U)$, the standard normalized form of LSF $(G)$ can be written as follows

$G(u)=Z\left(T^{-1}(u)\right)$

FORM works based on the approximation of Eq. (9) which is obtained by linearizing LSF in standard normal space at optimal point $\left(\mathrm{u}^{*}\right)$ (also named design point, most probable point, and beta point) which is determined by solving the following optimizing problem:

$u^{*}=\operatorname{argmin}\{\|u\| G(u)=0\}$

In other words, $\mathrm{u}^{*}$ is the minimum distance between the standard normalized form of $\operatorname{LSF}(\mathrm{G})$ and the origin in the standard normal space. The first term of the Taylor series for the normalized standard form of $\mathrm{Z}(\mathrm{x})$ is written as:

$G(u) \cong G_{1}(u)=\nabla G\left(u^{*}\right)\left(u-u^{*}\right)=$ $\left\|\nabla G\left(u^{*}\right)\right\|(\beta-\alpha u)$

where $\nabla G$ denotes gradient vector, $\alpha$ is normalized negative gradient row vector at design point (also called importance measurement) which is determined by Eq. (12), and $\beta$ is RI which is equal to $\beta=\alpha u^{*}$

$\alpha=-\nabla G\left(u^{*}\right) /\left\|\nabla G\left(u^{*}\right)\right\|$

The presented first-order approximation of the failure probability by FORM can be defined exactly by distance $\beta$ which is given by Eq. (13).

$P_{f} \cong P_{f 1}=\varphi(-\beta)$

FORM is applicable to a wide range of problems for calculating $\mathrm{P}_{\mathrm{f}}$. As the higher-order derivatives for the linear LSFs are zero, so FORM will yield adequately accurate results. Using FORM for nonlinear LSFs lead to a large difference between the approximated and the real results. Therefore, FORM does not apply to these problems, and alternative methods like SORM and MCS should be used instead [19].

\subsection{SORM}

The limit state could be nonlinear because of the nonlinear relationship between the random variables and the limit state function, or because of some variables being non-normal. Even a linear limit state in the original space becomes nonlinear when transformed to the standard normal space if any of the variables are non-normal. If the joint Probability Density Function (PDF) of the random variables decays rapidly as one moves away from the minimum distance point, then the above first-order estimate of the failure probability is quite accurate. If the decay of the 
joint PDF is slow, and the limit state is highly nonlinear, one must then use a higher-order approximation to compute the probability of failure [19].

\subsection{Sampling Method}

The most convenient and simplest method in reliability engineering is based on the generation of random numbers. The most famous sampling method is the "Monte-Carlo sampling" method which is the easiest and most useful method for reliability engineering [19]. In general, a numerical solution is necessary by one of two classes of methods: (i) Monte Carlo simulation and (ii) reliability methods.

The Monte Carlo simulation is conceptually simple. It is based on numerical sampling where a set of $X$ values are simulated from the corresponding probability distributions. These values are substituted in the function $Z(x)$ and the value of $g$ is compared to zero. The process is repeated a large number of times and count is kept of the ratio between the number of trials that lead to $Z \leq 0$ and the total number of trials. The ratio is used as an estimate of the desired probability value.

The reliability methods, which are developed in connection with structural reliability, provide approximate solutions for general probability integrals of the type presented in Eq. (7) over domains with smooth boundaries. The approximations involve a transformation of all parameter distributions into independent normal variables and the replacement of the function $\mathrm{Z}(\mathrm{x})$ by an approximate one. This allows using a special case for which an analytical solution for Eq. (7) exists. Of the two basic methods available, SORM provides a more accurate approximation than FORM because the function $\mathrm{Z}(\mathrm{x})$ is approximated by a second-order Taylor series expansion as opposed to the first-order expansion used in FORM. It is also possible to increase the accuracy of SORM results by using a simulation procedure which, by virtue of the SORM analysis, can be done very efficiently.

Each of the above approaches has its own pros and cons. The Monte Carlo method is conceptually simple and can easily deal with parameter dependencies, distribution truncations, and discrete random parameters. The main disadvantage is that, in most practical cases, a very large number of simulations (tens to hundreds of thousands, or even more for small probabilities) are needed and this tends to pose restrictions on the number of analyses that can be carried out. However, it must be noted that there are some recent developments in this method which may enhance its efficiency.

FORM and SORM have the advantage of being very efficient. Results can usually be obtained in a fraction of the time required for a Monte Carlo simulation. In addition, the analysis provides a measure of the sensitivity within the overall probability of failure to the different input variable parameters and their distributions as a byproduct. These methods have also been shown to provide sufficiently accurate solutions for small probabilities in a wide range of practical problems. Their disadvantage is that they use iterative numerical procedures which are not guaranteed to converge and occasionally cases may arise for which no solution cannot be found [15].

The reliability analysis has been computed by linking the buckling model to the reliability analysis software RT.

One of the main reasons for structural deterioration and pipe replacements is corrosion. Corrosion affects pipeline wall thickness.

To investigate the effect of changes in pipeline thickness, if there is no data available, the National Association of Corrosion Engineers (NACE) assumes the corrosion rate to be $0.4 \mathrm{~mm} /$ years [20].

\section{Results and Discussion}

This paper presents the reliability analysis to study and quantify the variations in the reliability index $(\beta)$ with the main parameters involved during the lateral buckling of submarine pipes caused by High-Pressure and High-Temperature (HPHT) conditions.

To assess the effect of geometric specifications of the pipelines on lateral bucking using Eq. (1), the required effective axial force to buckle is calculated for the uncertainties of parameters shown in Table 2 .

The uncertainties considered for the reliability assessment are summarized in Table 2.

The submarine pipeline has a lateral buckling if the resistance of the pipeline is smaller than the force exerted inside the pipeline due to HPHT.

The reliability methods are used as a mathematical tool to determine the probability of failure $\left(\mathrm{P}_{\mathrm{f}}\right)$ in some special conditions by considering uncertainties in both load and resistance parameters [21]. The uncertainties can be divided into epistemic and aleatoric [22].

The probability of failure $\left(P_{f}\right)$ and the reliability index $(\beta)$ can be calculated by FORM and other methods [23]. This research used three methods of FORM, SORM and sampling method to determine $P_{f}$ and $\beta$. The number of generated simulations in the Monte-Carlo sampling method is 40000 .

Figure 4 indicated three curves of PDF, CDF, and COV in the sampling method depicted in a single graph. To evaluate the effect of pipeline thickness and diameter on the reliability index in lateral buckling, the paper investigates the reliability index of the South Pars Gas Field pipeline. Figure 3 and Table 5 present $P_{f}$ and $\beta$. 


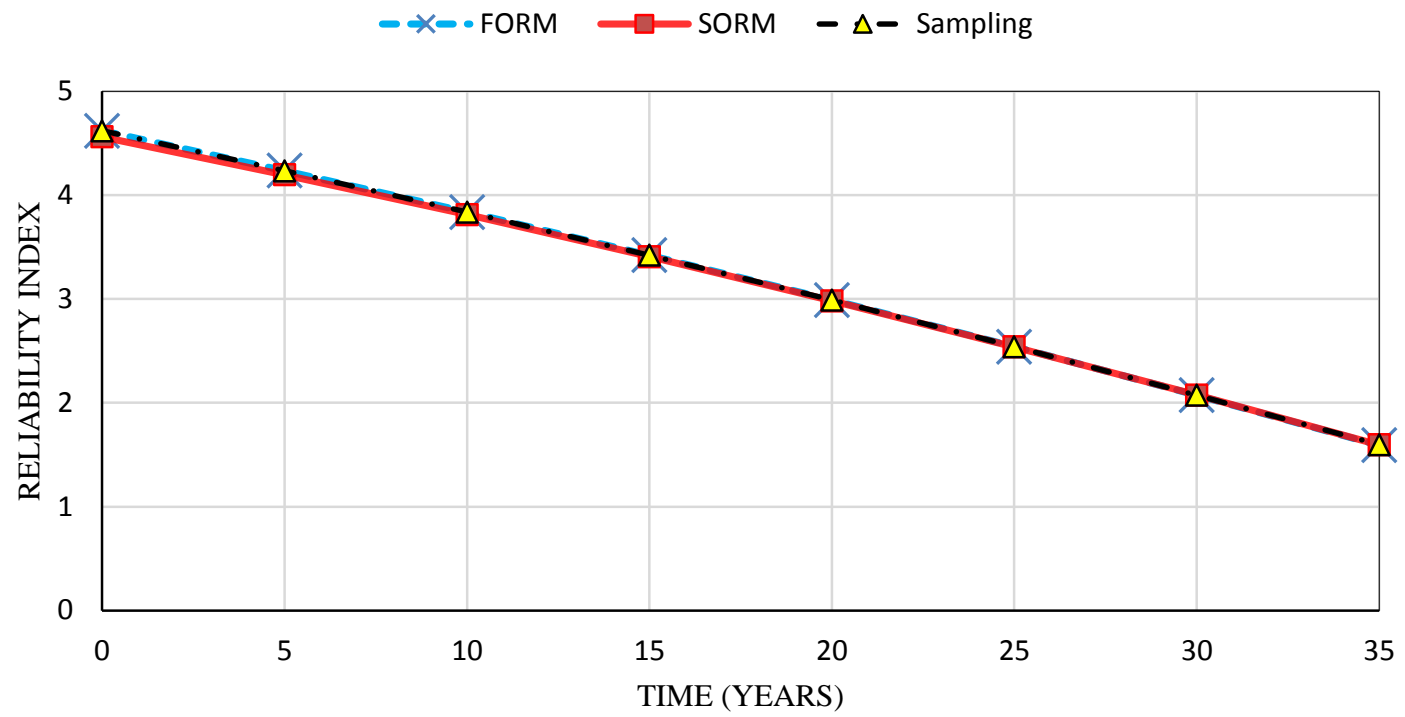

Figure 3. Reliability Index ( $\beta$ ) in FORM, SORM and Sampling Method

Table 5. The reliability index ( $\beta$ ) in FORM, SORM and Monte-Carlo (MC) sampling method

\begin{tabular}{lllll}
\hline Years & $\boldsymbol{t}[\boldsymbol{m}]$ & FORM & SORM & MC sampling \\
\hline 0 & 0.024 & 4.62046 & 4.56097 & 4.61976 \\
\hline 5 & 0.022 & 4.23732 & 4.19706 & 4.2354 \\
\hline 10 & 0.020 & 3.83802 & 3.81274 & 3.83759 \\
\hline 15 & 0.018 & 3.42214 & 3.40797 & 3.4215 \\
\hline 20 & 0.016 & 2.98946 & 2.98299 & 2.98944 \\
\hline 25 & 0.014 & 2.53997 & 2.53838 & 2.54042 \\
\hline 30 & 0.012 & 2.07405 & 2.07512 & 2.07399 \\
\hline 35 & 0.010 & 1.59244 & 1.59462 & 1.59689 \\
\hline
\end{tabular}

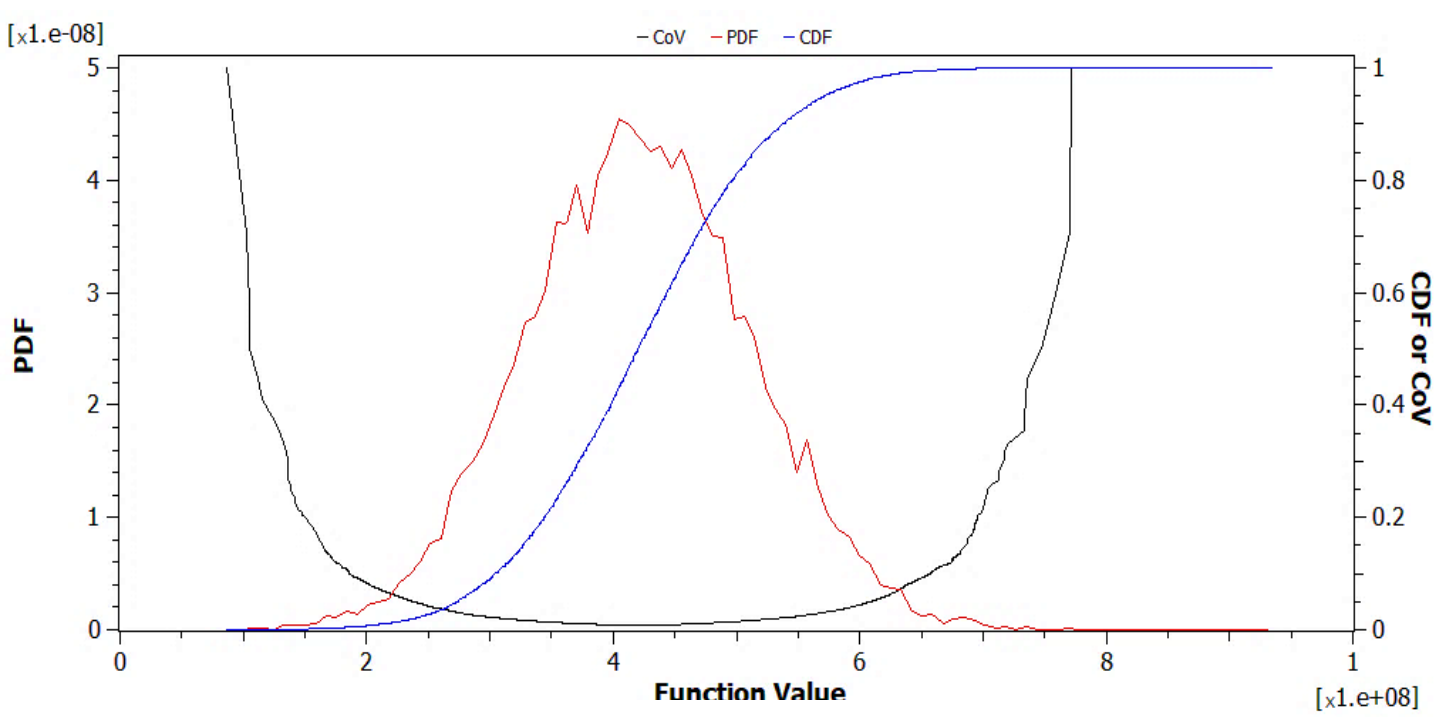

Figure 4. PDF, CDF and COV histogram in the sampling method 


\section{Conclusions}

In order to calculate the probability of failure of a pipeline due to the lateral buckling, three methods were used. Among these three methods, for a fixed condition, the SORM exhibits the lowest $\beta$ and the highest $P_{f}$. The results of the FORM and the sampling method are very close to each other.

It is also worth noting that the results of the different methods get closer to each other in 30 years over time. Finally, the results show that the pipeline, in the case of lateral buckling and corrosion, will be in a safe condition for up to 30 years after construction.

The results of this highly applied research can be the basis of considering other existing phenomena e.g. free span, local buckling and bursting simultaneously.

\section{List of Symbols}

$\begin{array}{ll}E & \text { Modulus of elasticity [GPa] } \\ t & \text { Wall thickness [m] } \\ t^{\prime} & \text { Pipe wall thickness due to corrosion [m] } \\ D & \text { Pipe diameter [m] } \\ S_{0} & \text { Effective axial compressive force, } \\ & \text { (compressive, }-; \text { tension, }+ \text { ) } \\ \Delta p_{i} & \text { Difference of internal pressure relative to } \\ \Delta T_{i} & \text { laying condition. } \\ A & \text { Difference between operating temperature } \\ A_{i} & \text { and installation temperature } \\ A_{s} & \text { Cross section area of pipe } \\ \vartheta & \text { Internal bore area of the pipe } \\ \alpha & \text { Cross-sectional area of the pipe } \\ F_{r e s i d u a l} & \text { Poisson's ratio } \\ Z & \text { Thermal expansion coefficient } \\ \omega & \text { Residual lay tension } \\ \mu_{l} & \text { The location on the pipe } \\ \mu_{a} & \text { Pipeline submerged unit weight } \\ L_{z} & \text { The lateral pipe-seabed friction coefficient } \\ k_{n} & \text { The axial pipe-seabed friction coefficient } \\ P_{f} & \text { Buckle length }\end{array}$

\section{References}

1- Rezazadeh, K., Zhu, L., Bai, Y., and Zhang, L., (2010), Fatigue Analysis of Multi-Spanning Subsea Pipeline, 29th International Conference on Ocean, Offshore and Arctic Engineering, Vol.5, Parts A and B, p.805-812.

2- Karampour, H. and Albermani, F., (2014), Experimental and numerical investigations of buckle interaction in subsea pipelines, Eng.Struct.66, p.8188.

3- Mustaffa, Z., (2011), System Reliability Assessment of Offshore Pipelines, Ph.D. thesis, University of Delft. 4- Bai, Q. and Bai, Y.,(2014),10 -Lateral Buckling and Pipeline Walking, in Subsea Pipeline Design, Analysis, and Installation, Q. Bai and Y. Bai, Eds. Boston: Gulf Professional Publishing, p. 221-253.
5- Karampour, H., Albermani, F. and Gross, J., (2013), On lateral and upheaval buckling of subsea pipelines, Eng.Struct.52, p.317-330.

6- Elsayed T, Leheta H and Yehya A (2012), "Reliability of subsea pipelines against lateral instability", Ships and Offshore Structures 7(2):229236.

7- Det Norske Veritas, (2013), Global Buckling of Submarine Pipelines, Structural Design due to High Temperature/High Pressure, DNV-RP-F110.

8- Carr, M., Sinclair, F., Bruton, D., (2006), Pipeline walking-Understanding the field layout challenges, and analytical solutions developed for the SAFEBUCK JIP. Houston: OTC 17945, Offshore Technology Conference.

9- Hobbs R.E. and Liang F., (1989), Thermal buckling of pipelines close to restraints, The Hague, The Netherlands: Offshore Mechanics and Arctic Engineering.

10- Det Norske Veritas, (2013), Submarine Pipeline Systems, DNV-OS-F101.

11- Rajeev, P., Robert, D.J., Thusyanthan, I. and Kodikara, J., (2013), Reliability analysis of upheaval bucking of offshore pipelines, Australian Geomechanics Journal, Vol.48, p.137-148.

12- Taheri, A., Shabani, M.M. and Daghigh, M., (2018), Investigation of the Effect of Local Buckling and VIV Fatigue on Failure Probability of Subsea Pipelines in Iranian South Pars Gas Field, IJMT, Vol.9, p.23-32.

13- Sopyan, Y., "An Overview to Lateral Buckling and its Mitigation", 2016. [Online]. Available: https://pipelinemaster.wordpress.com/category/ pipeline-design/global-buckling. [Accessed: 2018]. 14- Hobbs R.E., (1984), In-service buckling of heated pipelines, Transportation Engineering, Vol.110 (2), p.89-175.

15- Al-Sharif, A.M. and Preston, R., (1996), Structural Reliability Assessment of the Oman India Pipeline, OTC 8210, p.569-578.

16- Rathbone A., Abdel-Hakim M., Cumming G., Tørnes K., (2008), Reliability of lateral buckling formation from planned and unplanned buckle sites, Estoril, Portugal, OMAE2008- 57300, 27th International Conference on Offshore Mechanics and Arctic Engineering.

17- Brown G., Brunner M., Qi X., (2006), Lateral buckling reliability calculation methodology accounting for buckle interaction. Houston: OTC 17795, Offshore Technology Conference.

18- Carr M., Matheson I.C., Peek R., Saunders P., George N., (2004), Load and resistance modeling of the Penguins flowline under lateral buckling, $23^{\text {rd }}$ International Conference on Offshore Mechanics and Arctic Engineering. Canada: Vancouver; OMAE 2004-51192. Offshore Mechanics and Arctic Engineering. 
19- Shabani, M.M., (2017), Reliability Assessment of Existing Subsea Pipelines in the Persian Gulf, Master's thesis, Petroleum University of Technology.

20- Vanayi, H., Eslami, A., (2015), A Review of Inspection and Corrosion Rate Determination Methods, in Oil and Gas pipelines, $6^{\text {th }}$ Iranian Pipe and Pipeline Conference, Iran, Tehran, Beheshti International Conference Center.

21- Van den Abeele, F., Boël, F. and VandenBerghe, J.F., (2014), Structural Reliability of Free Spanning
Pipelines, Vol.3: Materials and Joining; Risk and Reliability

22- BOMEL Limited, (2001), Probabilistic Methods: Uses and Abuses in Structural Integrity, in Probabilistic methods: Uses and abuses in structural integrity, no. 398/2001.

23- Kroese, D.P. and Rubinstein, R.Y., (2017) Simulation and the Monte Carlo method, Third ed., John Wiley \& Sons, Inc., Hoboken, New Jersey. 